\title{
AN ALGORITHM FOR MATRIX EXTENSION AND WAVELET CONSTRUCTION
}

\author{
W. LAWTON, S. L. LEE AND ZUOWEI SHEN
}

\begin{abstract}
This paper gives a practical method of extending an $n \times r$ matrix $P(z), r \leq n$, with Laurent polynomial entries in one complex variable $z$, to a square matrix also with Laurent polynomial entries. If $P(z)$ has orthonormal columns when $z$ is restricted to the torus $\mathbf{T}$, it can be extended to a paraunitary matrix. If $P(z)$ has rank $r$ for each $z \in \mathbf{T}$, it can be extended to a matrix with nonvanishing determinant on $\mathbf{T}$. The method is easily implemented in the computer. It is applied to the construction of compactly supported wavelets and prewavelets from multiresolutions generated by several univariate scaling functions with an arbitrary dilation parameter.
\end{abstract}

\section{INTRODUCTION}

This note deals with matrix extension and a practical method for the construction of compactly supported wavelets and prewavelets from multiresolutions generated by several univariate compactly supported scaling functions with an arbitrary dilation parameter $m \in \mathbf{N}, m>1$. Such wavelets and prewavelets can have very small supports, a feature which may be important in numerical applications. The construction of wavelets and prewavelets from a multiresolution generated by a single univariate scaling function with the dilation parameter 2 was given in [12] and [1], respectively. It is well known that wavelet and prewavelet construction from a multiresolution generated by a finite number of compactly supported scaling functions can be reduced to the problem of extending a matrix with Laurent polynomial entries. This is widely studied in the case of wavelet and prewavelet construction in one and several dimensions from multiresolutions generated by one scaling function (see $[8,9,14,15])$ and by several scaling functions (see $[5,6,7,13])$. In practice it is necessary that the matrix extension be carried out constructively in order to obtain the wavelets explicitly. However, in the existing methods such an extension requires the knowledge of some intrinsic properties of the scaling functions. In this note, we shall give a constructive method which does not rely on the intrinsic properties of the scaling functions.

For a given finite set of compactly supported functions $\phi_{j}, j=1, \ldots, r$, let $V_{0}$ denote the closed shift invariant subspace generated by $\phi_{j}, j=1, \ldots, r$, i.e.,

Received by the editor February 15, 1994 and, in revised form, October 4, 1994 and January 30, 1995.

1991 Mathematics Subject Classification. Primary 41A15, 41A30, 15A54, 65D07, 65F30.

Key words and phrases. Wavelets, prewavelets, matrix extension, splines. 


$$
V_{0}:=\overline{\left\{\sum_{j=1}^{r} \sum_{k \in \mathbf{Z}} a_{j}(k) \phi_{j}(\cdot-k): a_{j} \in \ell_{0}(\mathbf{Z})\right\}},
$$

where $\ell_{0}$ is the space of finitely supported sequences. For $\nu \in \mathbf{Z}$, let

$$
V_{\nu}:=\left\{f\left(m^{\nu} \cdot\right): f \in V_{0}\right\} .
$$

We say that $\phi_{j}, j=1, \ldots, r$, are refinable if there are finitely supported sequences $p_{i, j}$ such that

$$
\phi_{i}(x)=\sum_{j=1}^{r} \sum_{k \in \mathbf{Z}} p_{i, j}(k) \phi_{j}(m x-k), \quad i=1, \ldots, r .
$$

The functions $\phi_{j}, j=1, \ldots, r$, are called refinable (or scaling) functions and $p_{i, j}$, $i, j=1, \ldots, r$, are called refinement masks.

If $\phi_{j}, j=1, \ldots, r$, are refinable and the set $\left\{\phi_{j}(\cdot-k): j=1, \ldots, r, k \in \mathbf{Z}\right\}$ forms an orthonormal (or Riesz) basis for $V_{0}$, then it is well known that $\left(V_{\nu}\right)_{\nu \in \mathbf{Z}}$ forms a multiresolution (cf. [9]). Compactly supported wavelets (prewavelets) are compactly supported functions $\psi_{j}, j=1, \ldots, r$, for which their shifts form an orthonormal (Riesz) basis of $W_{0}:=\left.V_{0}^{\perp}\right|_{V_{1}}$, the orthogonal complement of $V_{0}$ in $V_{1}$.

Let $\mathcal{R}[z]$ be the univariate Laurent polynomial ring over the complex field, and let $G_{n}(\mathcal{R})$ be the group of all $n \times n$ matrices over $\mathcal{R}[z]$ for which the determinants are nonvanishing on $\mathbf{C} \backslash\{0\}$. An $n \times n$ matrix $P(z)$ is called paraunitary if it is unitary on the unit circle $\mathbf{T}$. A diagonal matrix with diagonal entries of the form $z^{k}, k \in \mathbf{Z}$, is called a diagonal z-matrix. Clearly a diagonal $z$-matrix is paraunitary.

Let $\Phi:=\left(\phi_{1}, \phi_{2}, \ldots, \phi_{r}\right)^{T}$ and write (1.1) in matrix form

$$
\Phi(x)=\sum_{k \in \mathbf{Z}} P_{k} \Phi(m x-k)
$$

where

$$
P_{k}=\left(p_{i, j}(k)\right)_{i, j=1}^{r}
$$

Consider the $r \times r$ matrices

$$
P^{\ell}(z):=\frac{1}{\sqrt{m}} \sum_{k \in \mathbf{Z}} P_{\ell+k m} z^{k}, \quad \ell=0, \ldots, m-1,
$$

with Laurent polynomial entries, and form the $r \times m r$ block matrix

$$
P(z):=\left(P^{0}(z)|\cdots| P^{m-1}(z)\right),
$$

which is called a polyphase matrix. It is well known that if $\left\{\phi_{j}(\cdot-k): j=\right.$ $1, \ldots, r, k \in \mathbf{Z}\}$ forms an orthonormal basis of $V_{0}$, then $P(z) P(z)^{*}=I_{r}$ for all $z \in \mathbf{T}$. Compactly supported wavelets corresponding to the multiresolution generated by the scaling functions $\phi_{j}, j=1, \ldots, r$, can be constructed by extending the matrix $P$ to an $m r \times m r$ paraunitary matrix over $\mathcal{R}[z]$ in which the first $r$ rows are the matrix $P$. In $\S 2$, we give a constructive method to extend an arbitrary $r \times n$ matrix $P$, satisfying $P(z) P(z)^{*}=I_{r}$ for all $z \in \mathbf{T}$, to an $n \times n$ paraunitary matrix over $\mathcal{R}[z]$. This leads to a practical method for the construction of compactly supported wavelets from a finite number of scaling functions with an arbitary scaling parameter. In the construction of compactly supported wavelets, the refinement masks are usually known (in most cases the scaling functions are defined by their 
refinement masks via their Fourier transforms). Therefore, the above approach is quite natural.

If $\phi_{j}, j=1, \ldots, r$, form a Riesz basis of $V_{0}$, then $P(z)$ has rank $r$ for each $z \in \mathbf{T}$. If the refinement masks, and hence the polyphase matrix $P$, are available, the usual way of constructing the corresponding compactly supported prewavelets is to extend the polyphase matrix $P$ to an $m r \times m r$ matrix $Q$ over $\mathcal{R}[z]$ so that $Q(z)$ has rank $m r$ for all $z \in \mathbf{T}$. The standard Gram-Schmidt orthogonalization process is then applied to obtain the compactly supported prewavelets. Section 3 gives an algorithmic method to extend a general matrix. The method uses only elementary transformations and transformations by $z$-matrices and is easily implementable in the computer. Based on the matrix extension, we propose another approach in the construction of prewavelets. This approach gives prewavelets directly without using the Gram-Schmidt process after the matrix extension. The Gram-Schmidt process requires extra computing and enlarges the supports of the prewavelets. Our method usually gives prewavelets with shorter supports. This approach is also applicable if the refinement masks are not available, as in the case of the multiresolution generated by cardinal Hermite splines (see $\S 4$ ). The method is based on the sequence

$$
\tilde{p}_{i, j}(k):=m \int_{\mathbf{R}} \phi_{i}(x) \overline{\phi_{j}(m x-k)} d x
$$

where $\phi_{j}, j=1, \ldots, r$, are compactly supported scaling functions such that $\left\{\phi_{j}(\cdot-k): j=1, \ldots, r, k \in \mathbf{Z}\right\}$ forms a Riesz basis for $V_{0}$. The sequences $\tilde{p}_{i, j}$, $i, j=1, \ldots r$, are finitely supported, since $\phi_{j}, j=1, \ldots, r$, are compactly supported functions. They are readily computed from the scaling functions.

If $\left\{\tilde{\phi}_{j}(\cdot-k): j=1, \ldots, r, k \in \mathbf{Z}\right\}$ is the dual basis of $\left\{\phi_{j}(\cdot-k): j=\right.$ $1, \ldots, r, k \in \mathbf{Z}\}$, then

$$
\phi_{i}(x)=\sum_{j=1}^{r} \sum_{k \in \mathbf{Z}} \tilde{p}_{i, j}(k) \tilde{\phi}_{j}(m x-k), \quad i=1, \ldots, r .
$$

Equivalently,

$$
\Phi(x)=\sum_{k \in \mathbf{Z}} \widetilde{P}_{k} \widetilde{\Phi}(m x-k)
$$

where $\widetilde{\Phi}=\left(\tilde{\phi}_{1}, \ldots, \tilde{\phi}_{r}\right)^{T}$ and

$$
\widetilde{P}_{k}=\left(\tilde{p}_{i, j}(k)\right)_{i, j=1}^{r} .
$$

The corresponding $r \times r m$ block matrix

$$
\widetilde{P}(z):=\left(\widetilde{P}^{0}(z)|\cdots| \widetilde{P}^{m-1}(z)\right),
$$

where

$$
\widetilde{P}^{\ell}(z):=\sum_{k \in \mathbf{Z}} \widetilde{P}_{\ell+k m} z^{k}, \quad \ell=0, \ldots, m-1,
$$

will be called the dual polyphase matrix of $\Phi$. It has rank $r$ for all $z \in \mathbf{T}$, if $\phi_{j}$, $j=1, \ldots, r$, and their shifts form a Riesz basis for $V_{0}$. 
In $\S 3$, we shall give an algorithm to find an $(m-1) r \times m r$ matrix $Q^{\prime}(z)$ over $\mathcal{R}[z]$ such that the $m r \times m r$ matrix $\left(\frac{\widetilde{P}(z)}{Q^{\prime}(z)}\right)$ has rank $m r$ for all $z \in \mathbf{T}$ and

$$
\widetilde{P}(z) Q^{\prime}(z)^{*}=0 \quad \text { for } z \in \mathbf{T} \text {. }
$$

Suppose that

$$
Q^{\prime}(z)=:\left(Q^{0}(z)|\cdots| Q^{m-1}(z)\right)
$$

where each $Q^{\ell}, \ell=0, \ldots, m-1$, is an $r \times r$ matrix with Laurent polynomial entries

$$
\left(Q^{\ell}\right)_{i, j}(z)=: \sum_{k \in \mathbf{Z}} q_{i, j}(\ell+k m) z^{k}, \quad i, j=1, \ldots, r .
$$

Then the functions

$$
\psi_{i}(x):=\sum_{j=1}^{r} \sum_{k \in \mathbf{Z}} q_{i, j}(k) \phi_{j}(m x-k), \quad i=1, \ldots, m r-r,
$$

form a Riesz basis for $W_{0}$. Indeed, $\psi_{j}(\cdot-k) \perp \phi_{i}$ for $j=1, \ldots,(m-1) r, k \in$ $\mathbf{Z}, i=1, \ldots, r$, because of (1.3), (1.5) and (1.6). Hence, $\psi_{j}(\cdot-k) \in W_{0}, j=$ $1, \ldots,(m-1) r, k \in \mathbf{Z}$. Further, they form a Riesz basis of $W_{0}$ since $Q^{\prime}(z)$ has rank $(m-1) r$ for all $z \in \mathbf{T}$. This result in Hilbert space can be found in [10].

If $\phi_{j}, j=1, \ldots, r$, and their shifts form an orthonormal basis, then $\widetilde{P}=P$ and $P P^{*}=I_{r}$. In $\S 2$, we give a way to construct $Q^{\prime}$ such that $\left(\frac{P}{Q^{\prime}}\right)$ is a paraunitary matrix. With this $Q^{\prime}$, equation (1.6) gives the corresponding wavelets. The methods described above can also be extended to the construction of wavelets and prewavelets in Hilbert space.

Geronimo, Hardin and Massopust [4] have constructed two scaling functions whose shifts form an orthonormal basis of $V_{0}$. The corresponding wavelets were constructed by Donovan, Geronimo, Hardin and Massopust [3] and also by Strang and Strela [18]. Their works together with that of Goodman [5] are the main sources of motivation for this paper.

\section{Paraunitary matrix EXtension}

Let $A(z)$ be an $n \times 1$ matrix over $\mathcal{R}[z]$, and suppose that the smallest degree of its $j$ th component is $k_{j} \in \mathbf{Z}$, for $j=1,2, \ldots, n$. Let

$$
D(z):=\operatorname{diag}\left(z^{-k_{1}}, \ldots, z^{-k_{n}}\right) .
$$

Then $D A(z)$ is expressible in the form

$$
D A(z)=\mathbf{a}_{0}+\mathbf{a}_{1} z+\cdots+\mathbf{a}_{N} z^{N},
$$

where $\mathbf{a}_{j} \in \mathbf{C}^{n}, j=0, \ldots, N$, and $\mathbf{a}_{0}, \mathbf{a}_{N} \neq \mathbf{0}$.

Lemma 2.1. Let $A(z)=\sum_{j \in \mathbf{Z}} \mathbf{a}_{j} z^{j}$ be an $n \times 1$ matrix over $\mathcal{R}[z]$ with $\|A(z)\|=1$ for all $z \in \mathbf{T}$, where $\|A(z)\|$ denotes the Euclidean norm. Suppose that $j_{1}$ and $j_{2} \in \mathbf{Z}$ are respectively the lowest and highest degrees of $A(z)$. Then

$$
\sum_{j \in \mathbf{Z}}\left\langle\mathbf{a}_{j}, \mathbf{a}_{j}\right\rangle=1 \text { and }\left\langle\mathbf{a}_{j_{1}}, \mathbf{a}_{j_{2}}\right\rangle=0 .
$$


Proof. As is observed before the lemma, there exists a diagonal $z$-matrix $D$ such that each component of $D A$ is a polynomial. Hence, we need only to prove the case for which

$$
A(z)=\mathbf{a}_{0}+\mathbf{a}_{1} z+\cdots+\mathbf{a}_{N} z^{N}
$$

with $\|A(z)\|=1$ for $z \in \mathbf{T}$ and $\mathbf{a}_{0} \neq 0, \mathbf{a}_{N} \neq 0$. We note that on $\mathbf{T}$, the constant term of $\|A(z)\|^{2}$ is $\sum_{j=0}^{N}\left\langle\mathbf{a}_{j}, \mathbf{a}_{j}\right\rangle$ and the coefficient of the highest degree is $\left\langle\mathbf{a}_{0}, \mathbf{a}_{N}\right\rangle$. The results then follow from the fact that $\|A(z)\|^{2}=1$ for $z \in \mathbf{T}$.

Suppose that $D_{1}$ is a diagonal $z$-matrix such that

$$
D_{1} A(z)=\mathbf{a}_{0}^{(1)}+\mathbf{a}_{1}^{(1)} z+\cdots+a_{N}^{(1)} z^{N},
$$

where $\mathbf{a}_{j}^{(1)} \in \mathbf{C}^{n}, j=0, \ldots, N$, and $a_{0}^{(1)}, a_{N}^{(1)} \neq 0$. Let $U_{1}$ be a unitary matrix over $\mathbf{C}$ such that

$$
U_{1} \mathbf{a}_{0}^{(1)}=(\alpha, 0, \ldots, 0)^{T}, \quad \alpha \neq 0 .
$$

By Lemma 2.1, $\left\langle U_{1} \mathbf{a}_{0}^{(1)}, U_{1} \mathbf{a}_{N}^{(1)}\right\rangle=0$. Hence, the first entry $\left(U_{1} \mathbf{a}_{N}^{(1)}\right)_{1}$ of the vector $U_{1} \mathbf{a}_{N}^{(1)}$ is zero. Multiplying (2.1) by $U_{1}$ followed by an appropriate diagonal $z$-matrix $D_{2}$, we can express

$$
D_{2} U_{1} D_{1} A(z)=\mathbf{a}_{0}^{(2)}+\mathbf{a}_{1}^{(2)} z+\cdots+\mathbf{a}_{M}^{(2)} z^{M},
$$

where $\mathbf{a}_{0}^{(2)}, \mathbf{a}_{M}^{(2)} \neq \mathbf{0}$, and $M<N$. Furthermore,

$$
\left\|D_{2} U_{1} D_{1} A(z)\right\|=1, \quad z \in \mathbf{T},
$$

and $\left\langle\mathbf{a}_{0}^{(2)}, \mathbf{a}_{M}^{(2)}\right\rangle=0$, by Lemma 2.1. Repeating the above procedure gives a sequence of unitary matrices $U_{j}, j=1, \ldots, k$, over $\mathbf{C}$ and a sequence of diagonal z-matrices $D_{j}, j=1, \ldots, k$, such that

$$
U_{k} D_{k} U_{k-1} D_{k-1} \cdots D_{2} U_{1} D_{1} A(z)=(1,0, \ldots, 0)^{T}, \quad z \in \mathbf{T} .
$$

Let

$$
P(z):=U_{k} D_{k} \cdots U_{1} D_{1}(z), \quad z \in \mathbf{C} \backslash\{0\} .
$$

Then $P$ is paraunitary,

$$
P(z) A(z)=(1,0, \ldots, 0)^{T}, \quad z \in \mathbf{T},
$$

and for $z \in \mathbf{T}$,

$$
A(z)=P(z)^{*}(1,0, \ldots, 0)^{T},
$$

where $P(z)^{*}$ is the conjugate transpose of $P(z)$.

The relation (2.5) shows that the first column of the matrix $P(z)^{*}$ coincides with $A(z)$. Thus, $P(z)^{*}$ is a paraunitary extension of $A(z)$. The above algorithm can also be applied to extend an $n \times r$ matrix $A(z)$ with orthonormal columns and with entries in $\mathcal{R}[z]$, to a paraunitary matrix.

Theorem 2.1. Suppose that $A_{j}(z), j=1, \ldots, r$, are $n \times 1$ column vectors over $\mathcal{R}[z], r<n$, which are orthonormal on $\mathbf{T}$, and that $A(z):=\left(A_{1}(z)|\cdots| A_{r}(z)\right)$ is an $n \times r$ matrix over $\mathcal{R}[z]$. Then there exists an $n \times n$ paraunitary matrix $Q$ such that

$$
Q A(z)=\left(\frac{I_{r}}{0}\right)
$$


Furthermore,

$$
Q=Q_{r} Q_{r-1} \cdots Q_{1}
$$

where

$$
Q_{k}=\left(\begin{array}{c|c}
I_{k-1} & 0 \\
\hline 0 & P_{k}
\end{array}\right), \quad k=1, \ldots, r
$$

is an $n \times n$ paraunitary matrix and $P_{k}$ is an $(n-k+1) \times(n-k+1)$ paraunitary matrix of the form (2.3).

Proof. The observation before the theorem shows that there is a paraunitary matrix $Q_{1}$ of the form (2.3) such that

$$
Q_{1} A_{1}(z)=(1,0, \ldots, 0)^{T}, \quad z \in \mathbf{T} .
$$

Since $Q_{1}$ is a paraunitary matrix, it follows that for $i, j=1, \ldots, r$,

$$
\left\langle Q_{1} A_{i}(z), Q_{1} A_{j}(z)\right\rangle=\delta_{i, j}, \quad z \in \mathbf{T} .
$$

Hence, the first component $\left(Q_{1} A_{j}(z)\right)_{1}$ of $Q_{1} A_{j}(z)$ is zero for $z \in \mathbf{T}, j=2, \ldots, r$, and

$$
Q_{1} A(z)=\left(\begin{array}{c|ccc}
1 & 0 & \cdots & 0 \\
\hline 0 & A_{2}^{(2)}(z) & \cdots & A_{r}^{(2)}(z)
\end{array}\right), \quad z \in \mathbf{T},
$$

where $A_{j}^{(2)}(z), j=2, \ldots, r$, are $(n-1) \times 1$ matrices, with $\left\langle A_{i}^{(2)}(z), A_{j}^{(2)}(z)\right\rangle=\delta_{i, j}$ for all $z \in \mathbf{T}$.

Suppose that there are paraunitary matrices $Q_{1}, \ldots, Q_{k-1}, k<r$, such that

$$
Q_{k-1} \cdots Q_{2} Q_{1} A(z)=\left(\begin{array}{c|ccc}
I_{k-1} & 0 & \cdots & 0 \\
\hline 0 & A_{k}^{(k)}(z) & \cdots & A_{r}^{(k)}(z)
\end{array}\right), \quad z \in \mathbf{T},
$$

where $A_{j}^{(k)}(z), j=k, \ldots, r$, are $(n-k+1) \times 1$ matrices, with $\left\langle A_{i}^{(k)}(z), A_{j}^{(k)}(z)\right\rangle=\delta_{i, j}$, $z \in \mathbf{T}$. Let $P_{k}$ be an $(n-k+1) \times(n-k+1)$ paraunitary matrix of the form (2.3) such that

$$
P_{k} A_{k}^{(k)}(z)=(1,0, \ldots, 0)^{T}, \quad z \in \mathbf{T} .
$$

By a similar argument as above, we have for $i, j=k, \ldots, r$,

$$
\left\langle P_{k} A_{i}^{(k)}(z), P_{k} A_{j}^{(k)}(z)\right\rangle=\delta_{i, j}, \quad z \in \mathbf{T}
$$

and

Let

$$
\left(P_{k} A_{\ell}^{(k)}(z)\right)_{1}=0, \quad \ell=k+1, \ldots, r, z \in \mathbf{T}
$$

Then clearly $Q_{k}$ is paraunitary, and

$$
Q_{k}=\left(\begin{array}{c|c}
I_{k-1} & 0 \\
\hline 0 & P_{k}
\end{array}\right)
$$

$$
Q_{k} Q_{k-1} \cdots Q_{1} A(z)=\left(\begin{array}{c|ccc}
I_{k} & 0 & \cdots & 0 \\
\hline 0 & P_{k} A_{k}^{(k)}(z) & \cdots & P_{k} A_{r}^{(k)}(z)
\end{array}\right) .
$$

Finally, letting

we have

$$
Q:=Q_{r} Q_{r-1} \cdots Q_{1}
$$

$$
Q A(z)=\left(\frac{I_{r}}{0}\right), \quad z \in \mathbf{T} .
$$

This completes the proof. 
Remark 1. If $Q(z)$ satisfies (2.6), then $Q(z)^{*}$ is a paraunitary extension of $A(z)$. Further, the proof gives an algorithm for the construction of $Q(z)$. This can be applied in the construction of wavelets if the scaling functions and refinement masks are known.

Example 1. Consider the scaling functions $\phi_{1}$ and $\phi_{2}$ constructed by Geronimo, Hardin and Massopust [4]. They satisfy the matrix dilation equation

$$
\left(\begin{array}{c}
\phi_{1}(x) \\
\phi_{2}(x)
\end{array}\right)=\sum_{k=0}^{3} P_{k}\left(\begin{array}{c}
\phi_{1}(2 x-k) \\
\phi_{2}(2 x-k)
\end{array}\right),
$$

where

$$
\begin{aligned}
P_{0}=\frac{1}{10}\left(\begin{array}{cc}
6 & 8 \sqrt{2} \\
\frac{-\sqrt{2}}{2} & -3
\end{array}\right), & P_{1}=\frac{1}{10}\left(\begin{array}{cc}
6 & 0 \\
\frac{9 \sqrt{2}}{2} & 10
\end{array}\right), \\
P_{2}=\frac{1}{10}\left(\begin{array}{cc}
0 & 0 \\
\frac{9 \sqrt{2}}{2} & -3
\end{array}\right), & P_{3}=\frac{1}{10}\left(\begin{array}{cc}
0 & 0 \\
\frac{-\sqrt{2}}{2} & 0
\end{array}\right) .
\end{aligned}
$$

The corresponding polyphase matrix $P$ is given by

$$
P(z)=\frac{\sqrt{2}}{20}\left(\begin{array}{cccc}
6 & 8 \sqrt{2} & 6 & 0 \\
\frac{-1+9 z}{\sqrt{2}} & -3-3 z & \frac{9-z}{\sqrt{2}} & 10
\end{array}\right) .
$$

Let

$$
A(z)=\frac{\sqrt{2}}{20}\left(\begin{array}{cc}
6 & \frac{-1+9 z}{\sqrt{2}} \\
8 \sqrt{2} & -3-3 z \\
6 & \frac{9-z}{\sqrt{2}} \\
0 & 10
\end{array}\right)
$$

Symbolic computation using the above algorithm produces a $4 \times 4$ paraunitary matrix

$$
Q=\left(\begin{array}{cccc}
\frac{3}{5 \sqrt{2}} & \frac{4}{5} & \frac{3}{5 \sqrt{2}} & 0 \\
\frac{9 z^{-1}-1}{20} & \frac{-3\left(z^{-1}+1\right)}{10 \sqrt{2}} & \frac{-z^{-1}+9}{20} & \frac{1}{\sqrt{2}} \\
\frac{-9 z^{-1}+1}{20} & \frac{3\left(z^{-1}+1\right)}{10 \sqrt{2}} & \frac{z^{-1}-9}{20} & \frac{1}{\sqrt{2}} \\
\frac{9 z^{-1}+1}{10 \sqrt{2}} & \frac{3\left(-z^{-1}+1\right)}{10} & \frac{-z^{-1}-9}{10 \sqrt{2}} & 0
\end{array}\right)
$$

satisfying the relation

$$
Q A(z)=\left(\frac{I_{2}}{0}\right)
$$

The matrix $Q$ is a product of Householder matrices and a diagonal $z$-matrix. Indeed,

$$
Q=Q_{3} D Q_{2} Q_{1}
$$

where

$$
Q_{1}=\left(\begin{array}{cccc}
\frac{3}{5 \sqrt{2}} & \frac{4}{5} & \frac{3}{5 \sqrt{2}} & 0 \\
\frac{4}{5} & \frac{15-9 \sqrt{2}}{15-25 \sqrt{2}} & \frac{12}{15-25 \sqrt{2}} & 0 \\
\frac{3}{5 \sqrt{2}} & \frac{12}{15-25 \sqrt{2}} & \frac{41-15 \sqrt{2}}{50-15 \sqrt{2}} & 0 \\
0 & 0 & 0 & 1
\end{array}\right)
$$


and

$$
\begin{gathered}
Q_{2}=\left(\begin{array}{cccc}
1 & 0 & 0 & 0 \\
0 & \frac{6(-3+\sqrt{2})}{-20+6 \sqrt{2}} & \frac{1-3 \sqrt{2}}{-10+3 \sqrt{2}} & 0 \\
0 & \frac{1-3 \sqrt{2}}{-10+3 \sqrt{2}} & \frac{3(3-\sqrt{2})}{-10+3 \sqrt{2}} & 0 \\
0 & 0 & 0 & 1
\end{array}\right), \\
Q_{3}=\left(\begin{array}{cccc}
1 & 0 & 0 & 0 \\
0 & \frac{1}{2} & -\frac{1}{2} & \frac{1}{\sqrt{2}} \\
0 & -\frac{1}{2} & \frac{1}{2} & \frac{1}{\sqrt{2}} \\
0 & \frac{1}{\sqrt{2}} & \frac{1}{\sqrt{2}} & 0
\end{array}\right)
\end{gathered}
$$

$$
D=\operatorname{diag}(1,1 / z, 1,1) .
$$

The conjugate transpose $Q(z)^{*}$ of $Q(z)$ is a paraunitary extension of $A(z)$. Hence, the corresponding wavelets can be constructed using the last two columns of $Q^{*}$ and (1.6). The resulting wavelets are a symmetric and antisymmetric pair.

\section{Matrix EXTENSION}

In Theorem 2.1 it was assumed that the columns of the matrix $A(z)$ are orthonormal on $\mathbf{T}$, and we obtain a paraunitary matrix $Q$ such that

$$
Q A(z)=\left(\frac{I_{r}}{0}\right) \text {. }
$$

The conjugate transpose $Q(z)^{*}$ of $Q(z)$ is a paraunitary extension of $A(z)$. In this section we shall impose no conditions on $A(z)$ and consider the extension of $A(z)$ over $\mathcal{R}[z]$. The extension can be achieved in the same way as in the case of paraunitary extension. However, the transformations are accomplished by a sequence of elementary matrices and diagonal $z$-matrices instead of by unitary matrices.

Theorem 3.1. Let $A(z)$ be an $n \times 1$ matrix over $\mathcal{R}[z]$. Then there is a matrix $P \in G_{n}(\mathcal{R})$ such that

$$
P A(z)=(p(z), 0, \ldots, 0)^{T},
$$

where $p \in \mathcal{R}[z]$. Furthermore, $P$ is a product of elementary matrices and diagonal $z$-matrices.

Proof. Suppose $D_{1}(z)$ is a diagonal $z$-matrix such that

$$
D_{1} A(z)=\mathbf{a}_{0}^{(1)}+\cdots+\mathbf{a}_{N}^{(1)} z^{N}
$$

where $\mathbf{a}_{j}^{(1)} \in \mathbf{C}^{n}$ and $\mathbf{a}_{0}^{(1)}, \mathbf{a}_{N}^{(1)} \neq 0$. In practice, $D_{1}(z)$ is chosen so that the vector $\mathbf{a}_{0}^{(1)}$ has as many nonzero entries as possible, in order to speed up the process. Let $E_{1}$ be a product of elementary matrices which reduce the $n \times 2$ matrix $\left(\mathbf{a}_{0}^{(1)} \mid \mathbf{a}_{N}^{(1)}\right)$ to its "echelon form" with $E_{1} \mathbf{a}_{0}^{(1)}=(\alpha, 0, \ldots, 0)^{T}$, where $\alpha \neq 0$. Further, if $\mathbf{a}_{0}^{(1)}$ and $\mathbf{a}_{N}^{(1)}$ are linearly independent, we can choose $E_{1}$ so that the first entry of the vector $E_{1} \mathbf{a}_{N}^{(1)}$ is zero.

In the case

$$
E_{1} \mathbf{a}_{j}^{(1)}=\alpha_{j} E_{1} \mathbf{a}_{0}^{(1)}=\left(\alpha_{j}, 0, \ldots, 0\right)^{T}, \quad j=1, \ldots, N,
$$


then (3.1) holds with $P(z):=E_{1} D_{1}(z)$ and

$$
p(z):=1+\alpha_{1} z+\cdots+\alpha_{N} z^{N}, \quad z \in \mathbf{C} \backslash\{0\} .
$$

Otherwise, $E_{1} \mathbf{a}_{j}^{(1)}$ is not a multiple of $E_{1} \mathbf{a}_{0}^{(1)}$ for some $j$. Multiplying $E_{1} D_{1} A(z)$ by an appropriate diagonal $z$-matrix $D_{2}(z)$ gives

$$
D_{2} E_{1} D_{1} A(z)=\mathbf{a}_{0}^{(2)}+\cdots+\mathbf{a}_{N_{1}}^{(2)} z^{N_{1}}, \quad z \in \mathbf{C} \backslash\{0\},
$$

where $N_{1} \leq N$, and $\mathbf{a}_{0}^{(2)}, \mathbf{a}_{N_{1}}^{(2)} \neq 0$. Recall that $D_{2}$ is chosen so that the vector $\mathbf{a}_{0}^{(2)}$ has as many nonzero entries as possible. The case $N_{1}=N$ occurs if and only if $E_{1} \mathbf{a}_{N}^{(1)}=\alpha_{N} E_{1} \mathbf{a}_{0}^{(1)}$ for some $\alpha_{N} \neq 0$, i.e., $\mathbf{a}_{0}^{(1)}$ and $\mathbf{a}_{N}^{(1)}$ are linearly dependent. But in this case the vectors $\mathbf{a}_{0}^{(2)}$ and $\mathbf{a}_{N_{1}}^{(2)}$ are linearly independent.

Applying the above procedure, we can constructively find an invertible constant matrix $E_{2}$ which reduces $\left(\mathbf{a}_{0}^{(2)} \mid \mathbf{a}_{N_{1}}^{(2)}\right)$ to its "echelon form", with $E_{2} \mathbf{a}_{0}^{(2)}=$ $\left(\alpha^{\prime}, 0, \ldots, 0\right)^{T}, \alpha^{\prime} \neq 0$. Further, if $N_{1}=N$, then $\mathbf{a}_{0}^{(2)}$ and $\mathbf{a}_{N_{1}}^{(2)}$ are linearly independent. Hence, $E_{2}$ can be chosen such that $\left(E_{2} \mathbf{a}_{N_{1}}^{(2)}\right)_{1}=0$. Again, either (3.1) holds with $P:=E_{2} D_{2} E_{1} D_{1}$, or we can choose an appropriate diagonal $z$-matrix $D_{3}(z)$ such that

$$
D_{3} E_{2} D_{2} E_{1} D_{1} A(z)=\mathbf{a}_{0}^{(3)}+\cdots+\mathbf{a}_{N_{2}}^{(3)} z^{N_{2}}
$$

where $N_{2}<N$, and $\mathbf{a}_{0}^{(3)}, \mathbf{a}_{N_{2}}^{(3)} \neq 0$.

Since each entry of $D_{1} A(z)$ is a polynomial, and since the procedure reduces the degree of the entries, the process will stop after a finite number of steps. Hence, there is a sequence of invertible matrices $E_{j}, j=1, \ldots, k$, and a sequence of diagonal z-matrices $D_{j}, j=1, \ldots, k$, such that

$$
E_{k} D_{k} E_{k-1} D_{k-1} \cdots E_{1} D_{1} A(z)=(p(z), 0, \ldots, 0)^{T}
$$

for some $p \in \mathcal{R}[z]$. Therefore, (3.1) holds with

$$
P(z):=E_{k} D_{k} E_{k-1} D_{k-1} \cdots E_{1} D_{1}(z) \in G_{n}(\mathcal{R}), \quad z \in \mathbf{C} \backslash\{0\},
$$

as desired.

Direct application of Theorem 3.1 gives the following results.

Theorem 3.2. Suppose that $A_{j}(z), j=1, \ldots, r$, are $n \times 1$ column vectors over $\mathcal{R}[z], r<n$, and $A(z):=\left(A_{1}(z)|\cdots| A_{r}(z)\right)$ is an $n \times r$ matrix over $\mathcal{R}[z]$. Then there exists $Q \in G_{n}(\mathcal{R})$ such that

$$
Q A(z)=\left(\frac{B_{r}(z)}{0}\right)
$$

where $B_{r}(z)$ is an upper triangular $r \times r$ matrix over $\mathcal{R}[z]$. Furthermore,

$$
Q=Q_{r} Q_{r-1} \cdots Q_{1}
$$

where

$$
Q_{k}=\left(\begin{array}{c|c}
I_{k-1} & 0 \\
\hline 0 & P_{k}
\end{array}\right), \quad k=1, \ldots, r,
$$

and $P_{k}$ is an $(n-k+1) \times(n-k+1)$ matrix in $G_{n-k+1}(\mathcal{R})$ of the form (3.3). 
Note that the last $n-r$ rows of $Q$, which we denote by $Q_{r+1}^{\prime}, \ldots, Q_{n}^{\prime}$, form an $(n-r) \times n$ matrix

$$
Q^{\prime}:=\left(\begin{array}{c}
\frac{Q_{r+1}^{\prime}}{\vdots} \\
\hline Q_{n}^{\prime}
\end{array}\right)
$$

of rank $n-r$ for all $z \in \mathbf{C} \backslash\{0\}$. Further, by (3.4),

$$
A(z)^{*} Q^{\prime}(z)^{*}=0, \quad z \in \mathbf{C} \backslash\{0\} .
$$

If $A(z)=\left(A_{1}(z)|\cdots| A_{r}(z)\right)$ is an $n \times r$ matrix over $\mathcal{R}[z]$ of rank $r$ for each $z \in \mathbf{T}$ (or $z \in \mathbf{C} \backslash\{0\}$ ), then the $n \times n$ matrix $\left(A(z) \mid Q^{\prime}(z)^{*}\right)$ is an extension of $A$ satisfying (3.7) and of rank $n$ for all $z \in \mathbf{T}$ (or $z \in \mathbf{C} \backslash\{0\}$ ). That $\left(A(z) \mid Q^{\prime}(z)^{*}\right)$ is of rank $n$ follows from (3.7) and the fact that $A(z)$ and $Q^{\prime}(z)^{*}$ are of ranks $r$ and $n-r$, respectively, for all $z \in \mathbf{T}$ (or $z \in \mathbf{C} \backslash\{0\}$ ). This observation leads to

Corollary 3.1. Suppose that $A(z)=\left(A_{1}(z)|\cdots| A_{r}(z)\right)$ is an $n \times r$ matrix over $\mathcal{R}[z]$ of rank $r$ for each $z \in \mathbf{T}$ (or $z \in \mathbf{C} \backslash\{0\}$ ) and that $Q$ is a product of elementary matrices and diagonal $z$-matrices satisfying (3.4). If $Q_{r+1}^{\prime}, \ldots, Q_{n}^{\prime}$ are the last $n-r$ rows of $Q$ and

$$
Q^{\prime}(z):=\left(\frac{\frac{Q_{r+1}^{\prime}(z)}{\vdots}}{Q_{n}^{\prime}(z)}\right),
$$

then the matrix $\left(A(z) \mid Q^{\prime}(z)^{*}\right)$ is an extension of $A$ of rank $n$ for all $z \in \mathbf{T}$ (or $z \in \mathbf{C} \backslash\{0\})$ satisfying (3.7).

We note that this corollary can be applied directly in the construction of univariate prewavelets from a multiresolution generated by several scaling functions with an arbitrary dilation parameter $m \in \mathbf{Z}$. Since our proof of Theorem 3.1 is constructive and can be implemented in the computer step by step, this leads to a constructive method for the construction of prewavelets. We further remark that the Quillen-Suslin Theorem shows the existence of such an extension when the $n \times r$ matrix $A(z)$ over $\mathcal{R}[z]$ has rank $r$ for all $z \in \mathbf{C} \backslash\{0\}$. However, in the prewavelet construction, we usually assume that the scaling functions and their shifts form a Riesz basis of $V_{0}$, hence the corresponding $n \times r$ matrix $A$ is of rank $r$ only on $\mathbf{T}$. Further, our proof here for the univariate case is elementary and constructive.

We remark that further row operations may be performed on the last $n-r$ rows of the matrix $Q$ in Theorem 3.2 to obtain wavelets with desirable properties, like smallest support and symmetry. These operations preserve the relations (3.7). This is illustrated in the example in the following section.

\section{Cardinal Hermite spline wavelets}

For nonnegative integers $n \geq r$, let $\mathcal{S}_{2 n-1, r}(S)$ be the space of spline functions of degree $2 n-1$ defined on $\mathbf{R}$ with knots of multiplicity $r$ on the set $S$. The space $\mathcal{S}_{2 n-1, r}(\mathbf{Z})$ has a basis comprising functions $\phi_{\ell}, \ell=1, \ldots, r$, with minimal supports. The functions are called cardinal Hermite $B$-splines and are uniquely determined by the condition that they vanish outside $[0,2 n-2 r+2]$ and that they satisfy the Hermite interpolating conditions

$$
\phi_{\ell}^{(k-1)}(\nu)=c_{\nu} \delta_{\ell, k}, \quad \nu=1, \ldots, 2 m-2 r+1, k, \ell=1, \ldots, r,
$$


where $c_{\nu}, \nu=1, \ldots, 2 n-2 r+1$, are the coefficients of the generalized EulerFrobenius polynomial

$$
\Pi_{2 n-1, r}(\lambda)=\sum_{\nu=0}^{2 n-2 r} c_{\nu+1} \lambda^{\nu},
$$

which may be defined as the minor of order $(2 n-r) \times(2 n-r)$ obtained by removing the first $r$ rows and last $r$ columns of the matrix

$$
F_{2 n-1}(\lambda):=\left(\left(\begin{array}{l}
k \\
\ell
\end{array}\right)-\lambda \delta_{k, \ell}\right)_{k, \ell=0}^{2 n-1}
$$

(see $[17,11])$.

Let $V_{0}$ be the shift invariant subspace generated by $\phi_{\ell}, \ell=1, \ldots, r$. In $[6]$ it was shown that the shifts $\phi_{\ell}(x-k), \ell=1, \ldots, r, k \in \mathbf{Z}$, form a Riesz basis of $V_{0}$. Let

$$
V_{\nu}:=\left\{f\left(2^{\nu} x\right): f \in V_{0}\right\}, \quad \nu \in \mathbf{Z} .
$$

Then $V_{\nu} \subset V_{\nu+1}, \nu \in \mathbf{Z}$, and $\left\{V_{\nu}\right\}_{\nu \in \mathbf{Z}}$ is a multiresolution of $L^{2}(\mathbf{R})$ of multiplicity $r$.

Since the scaling functions are explicitly known, their dual polyphase matrix $\widetilde{P}(z)$ can be computed using (1.2), and hence the method of $\S 3$ is applicable for the construction of the corresponding prewavelets. The following example shows the construction of cubic cardinal Hermite spline wavelets from two interpolatory cubic Hermite scaling functions. This corresponds to the case $m=r=2$.

Example 2. The scaling functions $\phi_{1}, \phi_{2}$ are supported on $[0,2]$, and are given by

$$
\begin{gathered}
\phi_{1}(x)= \begin{cases}3 x^{2}-2 x^{3}, & 0 \leq x \leq 1 \\
-4+12 x-9 x^{2}+2 x^{3}, & 1 \leq x \leq 2,\end{cases} \\
\phi_{2}(x)= \begin{cases}-x^{2}+x^{3}, & 0 \leq x \leq 1 \\
-4+8 x-5 x^{2}+x^{3}, & 1 \leq x \leq 2\end{cases}
\end{gathered}
$$

They are $C^{1}$ on $\mathbf{R}$ and satisfy the interpolation conditions

$$
\begin{array}{ll}
\phi_{1}(\nu+1)=\delta_{0 \nu}, & \phi_{2}^{\prime}(\nu)=0, \quad \nu \in \mathbf{Z}, \\
\phi_{2}^{\prime}(\nu+1)=\delta_{0 \nu}, & \phi_{2}(\nu)=0, \quad \nu \in \mathbf{Z} .
\end{array}
$$

The dual polyphase matrix for $\phi_{1}, \phi_{2}$ is

$$
\begin{aligned}
6720 \widetilde{P}(z)=\left(\begin{array}{cc}
1680+1680 z & 152-152 z \\
-364+364 z & -20-20 z
\end{array}\right. \\
\left.\quad \begin{array}{cc}
138 z^{-1}+3084+138 z & 34 z^{-1}-34 z \\
-41 z^{-1}+41 z & -10 z^{-1}+64-10 z
\end{array}\right) .
\end{aligned}
$$

Let $A(z):=\widetilde{P}(z)^{*}, z \in \mathbf{T}$. The method of $\S 3$ gives a $4 \times 4$ matrix $Q$ satisfying the relation $Q A=B$, where

$$
B=\left(\begin{array}{cc}
304 & \left(194 z^{-1}-794\right) / 15 \\
0 & 1358 / 19 \\
0 & 0 \\
0 & 0
\end{array}\right)
$$


Further row operations on the last two rows of $Q$ produce another matrix, which we shall still denote by $Q=\left(Q_{i j}\right)$ and which still satifies $Q A=B$. The entries of $Q$ are

$$
\begin{aligned}
Q 11(z)= & \frac{19}{210}, Q_{12}(z)=1, Q_{13}(z)=0, Q_{14}(z)=0 \\
Q_{21}(z)= & \frac{23273695 z}{290116016}-\frac{102640647 z^{2}}{290116016}, \\
Q_{22}(z)= & \frac{628606657 z}{290116016}+\frac{1361706661 z^{2}}{290116016} \\
Q_{23}(z)= & \frac{679 z}{2482}, \quad Q_{24}(z)=\frac{269563 z}{58444} \\
Q_{31}(z)= & -\frac{5261}{3076}+\frac{2185 z}{1538}-\frac{5261 z^{2}}{3076} \\
Q_{32}(z)= & -\frac{34895}{1538}+\frac{34895 z^{2}}{1538}, \\
Q_{33}(z)= & 1+z, \quad Q_{34}(z)=-\frac{16133}{769}+\frac{16133 z}{769}, \\
Q_{41}(z)= & -\frac{179219}{204554}+\frac{179219 z^{2}}{204554}, \\
Q_{42}(z)= & -\frac{679449}{58444}-\frac{139611 z}{29222}-\frac{679449 z^{2}}{58444}, \\
Q_{43}(z)= & 1-z, \quad Q_{44}(z)=-\frac{186150}{14611}-\frac{186150 z}{14611}
\end{aligned}
$$

Let $Q^{\prime}$ be the $2 \times 4$ matrix comprising the last two rows of $Q$. Then $\left(A(z) \mid Q^{\prime}(z)^{*}\right)$ is an extension of $A(z)$ of rank 4 satisfying (3.7). If we write

$$
Q^{\prime}=\left(Q^{0} \mid Q^{1}\right)=\left(\begin{array}{ll|ll}
Q_{11}^{0} & Q_{12}^{0} & Q_{11}^{1} & Q_{12}^{1} \\
Q_{21}^{0} & Q_{22}^{0} & Q_{21}^{1} & Q_{22}^{1}
\end{array}\right)
$$

and define $q_{i, j}(k), i, j=1,2, k=1, \ldots, 4$, by

$$
Q_{i, j}^{\ell}(z)=: \sum_{k=0}^{2} q_{i, j}(\ell+2 k) z^{k}, \quad \ell=0,1
$$

the corresponding wavelets $\left\{\psi_{1}, \psi_{2}\right\}$ which generate a Riesz basis for $W_{0}$ are given by

$$
\psi_{1}(x)=\sum_{k=0}^{4} q_{1,1}(k) \phi_{1}(2 x-k)+q_{1,2}(k) \phi_{2}(2 x-k)
$$

and

$$
\psi_{2}(x)=\sum_{k=0}^{4} q_{2,1}(k) \phi_{1}(2 x-k)+q_{2,2}(k) \phi_{2}(2 x-k),
$$

where the coefficients $q_{1, j}(k)$ and $q_{2, j}(k)$, up to 10 significant figures, are given in Tables 1 and 2 . 
TABLE 1 . Coefficients $q_{1, j}(k)$

\begin{tabular}{|c|c|c|}
\hline$k \backslash j$ & 1 & 2 \\
\hline 0 & -1.710338101 & -22.68855657 \\
1 & 1 & -20.97919376 \\
2 & 1.420676202 & 0 \\
3 & 1 & 20.97919376 \\
4 & -1.710338101 & 22.68855657 \\
\hline
\end{tabular}

TABLE 2. Coefficients $q_{2, j}(k)$

\begin{tabular}{|c|c|c|}
\hline$k \backslash j$ & 1 & 2 \\
\hline 0 & -0.8761451744 & -11.62564164 \\
1 & 1 & -12.74040107 \\
2 & 0 & -4.777599069 \\
3 & -1 & -12.74040107 \\
4 & 0.8761451744 & -11.62564164 \\
\hline
\end{tabular}

Figures 1 and 2 show the graphs of $\psi_{1}$ and $\psi_{2}$, respectively. Note that $\psi_{1}$ is symmetric and $\psi_{2}$ is antisymmetric about $\frac{3}{2}$, and both have support on $[0,3]$.

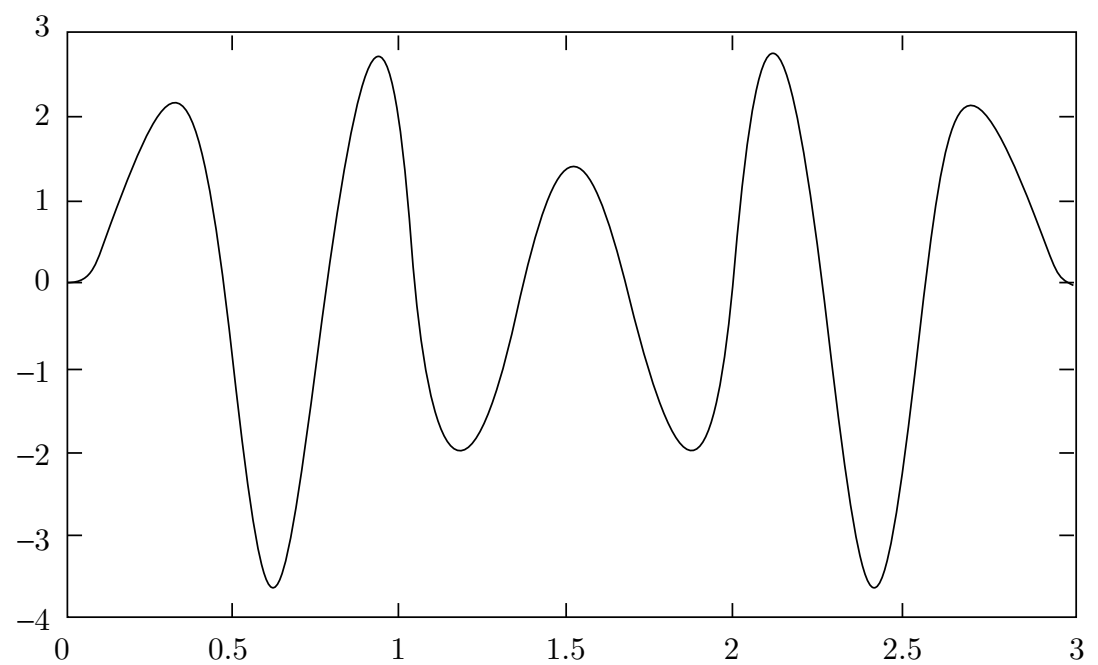

FIGURE 1. Graph of $\psi_{1}$ 


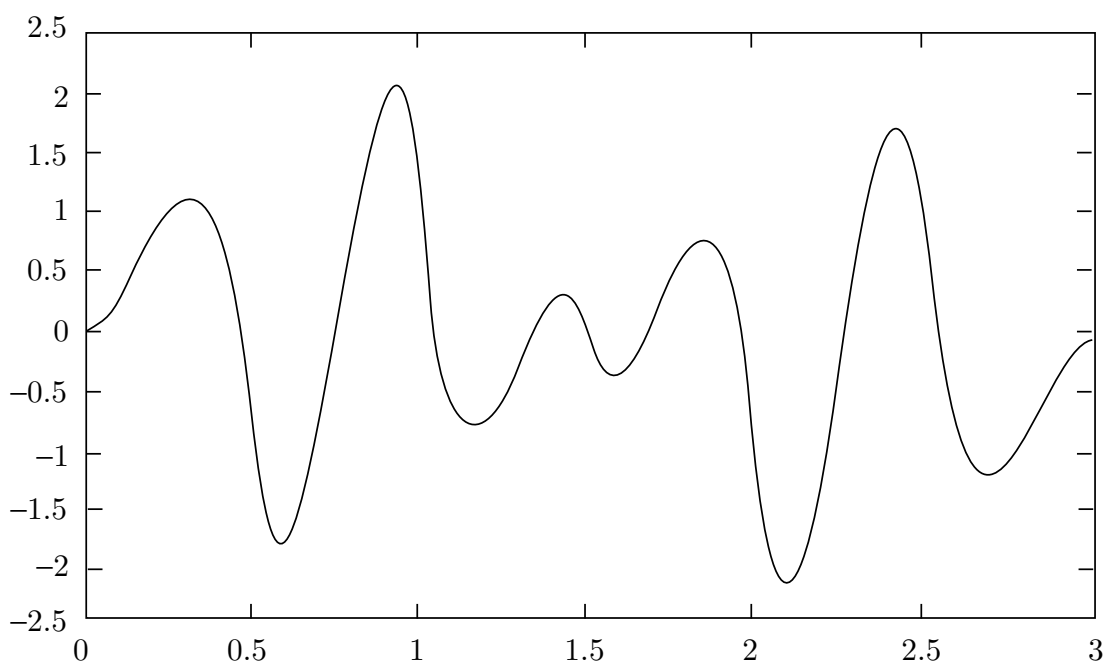

FiguRE 2. Graph of $\psi_{2}$

\section{REFERENCES}

1. Chui, K. Charles and Wang, Jian-zhong, On compactly supported spline wavelets and a duality principle, Trans. Amer. Math. Soc. 330 (1992), 903-915. MR 92f:41020

2. Daubechies, L., Orthonormal bases of compactly supported wavelet, Comm. Pure and Appl. Math. 41 (1988), 909-996. MR 90m:42039

3. Donovan, G., Geronimo, J. S., Hardin, D. P. and Massopust, P. R., Construction of orthogonal wavelets using fractal interpolation functions, SIAM J. Math. Anal., to appear

4. Geronimo, J. S., Hardin, D. P. and Massopust, P. R., Fractal functions and wavelet expansions based on several scaling functions, J. Approx. Theory 78 (1994), 373-401. CMP 94:17

5. Goodman, T. N. T., Interpolatory Hermite spline wavelets, J. Approx. Theory 78 (1994), 174-189. CMP 94:15

6. Goodman, T. N. T., Lee S. L. and Tang W. S., Wavelets in wandering subspaces, Trans. Amer. Math. Soc. 338 (1993), 639-654. MR 93j:42017

7. Goodman, T. N. T. and Lee S. L., Wavelets of multiplicity r, Trans. Amer. Math. Soc. 342 (1994), 307-324. MR 94k:41016

8. Jia, R. Q. and C. A. Micchelli, Using the refinement equation for the construction of prewavelets II: Powers of two, Curves and Surfaces (P. J. Laurent, A. Le Méhauté and L. L. Schumaker, eds.), Academic Press, New York, 1991, pp. 209-246. MR 93e:65024

9. Jia, R. Q. and Z. Shen, Multiresolution and Wavelets, Proc. Edinburgh Math. Soc. 37 (1994), 271-300. CMP 94:14

10. Lee, S. L., W. S. Tang, Tan H. H., Wavelet bases for a unitary operator, Proc. Edinburgh Math. Soc. 38 (1995), 233-260. CMP 95:13

11. Lee, S. L., B-splines for cardinal Hermite interpolation, Linear Algebra Appl. 12 (1975), 269-280. MR 52:3798

12. Mallat, S., Multiresolution approximations and wavelet orthonormal bases of $L^{2}(\mathbf{R})$, Trans. Amer. Math. Soc. 315 (1989), 69-87. MR 90e:42046

13. Micchelli, C. A. Using the refinement equation for the construction of prewavelets VI: Shift invariant subspaces, Approximation Theory, Spline Functions and Applications, S. P. Singh (ed.), Kluwer Academic Publishers, 1992, 213-222. MR 94f:42045

14. Riemenschneider, S. D. and Z. Shen, Box splines, cardinal series and wavelets, in "Approximation Theory and Functional Analysis", C. K. Chui, ed., Academic Press, New York, 1991, pp. 133-149. CMP 91:07

15. Riemenschneider, S. D. and Z. Shen, Wavelets and prewavelets in low dimensions, J. Approx. Theory 71 (1992), 18-38. MR 94d:42046 
16. Schoenberg, I.J., Cardinal spline interpolation, CBMS-NSF Series in Appl. Math., Vol. 12, SIAM Publ., Philadelphia, 1973. MR 54:8095

17. Schoenberg, I. J. and Sharma, A., Cardinal interpolation and spline functions $V$. The $B$ splines for cardinal Hermite interpolation, Linear Algebra Appl. 7 (1973), 1-42. MR 57:17085

18. Strang, G. and Strela V., Short Wavelets and Matrix Dilation Equations, preprint.

Institute of Systems Science, National University of Singapore, Heng Mui Keng Terrace, Kent Ridge, Singapore 0511

E-mail address, W. Lawton: wlawton@iss.nus.sg

Department of Mathematics, National University of Singapore, 10 Kent Ridge CresCENT, SINGAPORE 0511

E-mail address, S. L. Lee: matleesl@math.nus.sg

E-mail address, Z. Shen: matzuows@math.nus.sg 